Original Research Paper

\title{
Bifurcation and Stability Analysis of a Food Web in a Chemostat
}

\author{
S.M. Sohel Rana \\ Department of Mathematics, University of Dhaka, Dhaka-1000, Bangladesh
}

\author{
Article history \\ Received: 17-11-2014 \\ Revised: $15-05-2016$ \\ Accepted: 13-10-2016 \\ E-mail: srana.mthdu@gmail.com
}

\begin{abstract}
In this study, a classical model describing a food web in a chemostat involving three species competing for non-reproducing, growth rate-limiting nutrient in which one of the competitors predates on one of the other competitors is considered. Quantitative analyses of non-negativity and boundedness of solution trajectories, dissipativity, behavior around equilibria, global stability and persistence of the model equations are analyzed. We present the global stability of equilibria by constructing a Lyapunov function. Hopf bifurcation theory is applied.
\end{abstract}

Keywords: Chemostat, Food Web, Global Stability, Hopf Bifurcation, Dissipative

\section{Introduction}

In microbiology and population biology, the laboratory device chemostat extensively uses as research technique to culture microorganisms continuously under nutrient limitation in a controlled environment in order to study the general properties of its growth and interaction. Since one can measure the control parameter easily, the device has various applications in ecology and population biology. It can be viewed as a simple lake system in ecology while it serves as a laboratory bioreactor in chemical engineering used for investigations in genetically altered cell. As for example, the prey (bacteria) consumes nutrient (waste) while the predator (ciliates) feeds on the prey in waste water treatment process. It is of mathematical interest to construct models with chemostat. The dynamics of chemostat model with nutrient uptake is of Monod kinetics play an important role in population ecology. After the first introduction of chemostat the researchers have paid their attention to develop mathematical theories of models in it. Qualitative analyses of predator-prey models in chemostat both from the experimental and the modeling aspect describe by set of differential equations were studied by many authors (Aris and Humphrey, 1977; Butler and Wolkowicz, 1985; Drake and Tsuchiya, 1976; Huang and Zhu, 2005; Hsu et al., 1977; Jost et al., 1973a; Li, 1999; Tsuchiya et al., 1972; Wolkowicz and Lu, 1992; Waltman et al., 1980). For detailed descriptions and the complete mathematical theories for understanding models in a chemostat, we refer Smith and
Waltman (1995). Moreover, using chemostat the model describing predation-prey interaction like food chain and food web and their analyses are more interesting problems in the field of mathematical biology (Alhumazi and Ajbar, 2005; Butler and Wolkowicz, 1986; El-Owaidy and Moniem, 2003; Li and Kuang, 2000; Pavlou, 1985; Zhu et al., 2002).

The interactions between species or organisms by using chemostat studied in ecology are predation and competition. In predation, one of the individuals serves as a prey for other individuals but in competition, the individuals compete for the same resources (food supply, water, etc). Competition can occur between individuals of different species or among individuals of the same species. The system comprise of the two interactions (predation and competition) is a food chain or food web. A chemostat type food chain model had been studied in (Tsuchiya et al., 1972; Jost, et al., 1973a; Drake and Tsuchiya, 1976; Butler et al., 1983) and related experiments are described in (Jost et al., 1973b; Drake and Tsuchiya, 1976). They used MichaelisMenten type functional responses and discharge rates for the both population are equal to the washout rate of the chemostat. In the study (Butler et al., 1983), the authors obtained a global result of their model that show all competing predators coexist which circumvented competitive exclusion principle. But the authors (Li and Kuang, 2000) relaxed the assumptions that the functional responses are general monotone functions and discharge rates for the both population are distinct. They studied a predator-prey food chain model in a chemostat, where 
predator feeds on the prey and the prey consumes the nutrient. The authors (El-Owaidy and Moniem, 2003) also studied the same model with the extension that the predator feeds on the prey and the nutrient simultaneously. The paper (Alhumazi and Ajbar, 2005) examined a model describing the dynamic behavior of predator-prey interaction with general growth rates having no restriction on its forms. In general, it is difficult to analyze and study a complete dynamics for tri (or higher)-tropic level food chain models concern with its solutions. Consequently, the persistence or uniformly persistence analysis has been applied to describe the situation when all interacting individuals coexist in a given system. A tri-tropic level food-chain model is considered in (Chiu and Hsu, 1998). They restricted their attentions to study when the prey grows logistically in the absence of predator and the functional responses of predators are of Holling's type II and established the criterion where the top-predator goes extinct globally. The dynamics of a three-level food chain models that incorporate either Michaelis-Menten or general monotone response functions for all trophic levels had been examined by many researchers (Boer et al., 1998; El-Sheikh and Mahrouf, 2005; Freedman and Ruan, 1992; Freedman and Waltman, 1977; Hastings and Powell, 1991; Klebanoff and Hastings, 1994; Ruan and Freedman, 1991; Al-Sheikh, 2008). Bifurcation techniques also used in several studies to explore whether it is possible the coexistence of all trophic levels (Boer et al., 1998; El-Sheikh and Mahrouf, 2005; Freedman and Ruan, 1992; Al-Sheikh, 2008). Persistence analyses of models were carried out in (Butler et al., 1986; Freedman and Waltman, 1984; Ruan and Freedman, 1991).

A food web model describing predator -prey interaction consist of one predator and two competitors with a growth rate- limiting nutrient in which the predator feeds on one of the preys only had been theoretically investigated by (Butler and Wolkowicz, 1986; Wolkowicz, 2006). In (Butler and Wolkowicz, 1986), they considered the general monotone response functions, but in (Wolkowicz, 2006), the response functions that satisfy the law of mass action were assumed. On the other hand, a food web in a chemostat that incorporates one predator and two preys in which the predator predates on both of the preys and the preys consume the nutrient had been studied theoretically by (Nasrin and Rana, 2011), experimentally in lab by (Jost et al., 1973b) and numerically by (Vayenas and Pavlou, 1999). In all of their models, they employed either general monotone functions or Michaelis-Menten kinetics to describe uptake function of nutrient and growth of competitor (prey). In this study, we consider a model in a chemostat comprises of a predator individual and two competitors one of which serves as prey. We restrict our attention to examine the dynamics of the model (so called food web) where the competitors consume the nutrient and the predator feeds on one of the competitors and nutrient in the chemostat that incorporates general response functions. Our system can be viewed as resulting from the food-chain system (El-Owaidy and Moniem, 2003) by adding another competitor or from the competition system $(\mathrm{Li}, 1999)$ by adding a competitor preying upon one of the competitors. In this study we confine our interest to explore the criterion for global stability and persistence of the model for which all the individuals coexists in a steady state for all future time. This model can be viewed as a system experimentally in lab by considering two bacteria as prey, a protozoan as predator and glucose as the limiting nutrient in the chemostat.

This paper is organized as follows. The food web model with general monotonic functional responses is described in section 2 . In section 3 , the basic properties (boundedness and dissipativity of the system and the existence and the local stability of equilibrium points) are given. Section 4 deals with the global stability of the system around the equilibria. In section 5, Hopf bifurcation theory applied to the system is presented. Finally in section 6, we carried out a short discussion.

\section{Model Equations}

Suppose that the concentration of nutrient and populations are all continuous functions of time. Let the concentration of nutrient, the competing populations and the predator population be denoted by $s(t), x_{1}(t), x_{2}(t)$ and $y(t)$ respectively at time $t$. The model equations take the following form (a system of differential equations):

$$
\begin{aligned}
& s^{\prime}(t)=\left(s^{0}-s(t)\right) D-\sum_{i=1}^{2} \frac{x_{i}(t) p_{i}(s(t))}{\eta_{i}}-\frac{y(t) p_{3}(s(t))}{\eta_{3}} \\
& x_{1}^{\prime}(t)=x_{1}(t)\left(-D+p_{1}(s(t))\right)-\frac{1}{\gamma} y(t) q\left(x_{1}(t)\right) \\
& x_{2}{ }^{\prime}(t)=x_{2}(t)\left(-D+p_{2}(s(t))\right) \\
& y^{\prime}(t)=y(t)\left(-D+p_{3}(s(t))+q\left(x_{1}(t)\right)\right)
\end{aligned}
$$

with $s(0)=s_{0} \geq 0, x_{i}(0)=x_{i 0} \geq 0, i=1,2, y(0)=y_{0} \geq 0$ for $s^{0}>0$

In the system (1), $s^{0}$ denotes the input nutrient concentration, $p_{i}(s)$ represent the $i$ th competitor's specific per capita growth rate, $\eta_{i}$ are the yield constants and $D$ is the washout rate of the chemostat. In the model equations (1), the population $x_{1}(t)$ can be treated as both a prey and a competitor. Here, $q\left(x_{1}\right)$ is the predator's growth rate on prey population and $\gamma$ is the yield factor of predator feeding on prey.

The basic assumptions on the response functions $p_{i}$ and $q$ of the model (1) are: 


$$
p_{i}, q: \mathrm{R}_{+} \rightarrow \mathrm{R}_{+}
$$

where, $p_{i}, q$ are continuously differentiable; $p_{i}{ }^{\prime}(s) \geq 0$ for all $s \in \mathrm{R}_{+} ; q^{\prime}\left(x_{1}\right) \geq 0$ for all $x_{1} \in \mathrm{R}_{+} ; p_{i}(0)=0$ and $q(0)=0$.

The model (1) describes four-level predator-prey food web in a chemostat with non-reproducing, growth rate-limiting nutrient, constant dilution rate and input nutrient concentration, negligible death rates and instantaneous growth rates adjustment due to changes in the nutrient concentration. Furthermore, we assume that the uptake rate of nutrient is proportional to conversion rate of prey and similarly uptake rate of prey is proportional to conversion rate of predator. In model (1), the two competitor populations compete exclusively for a single, growth rate-limiting nutrient and the predator population feeds exclusively on one of the competitor population as prey that would be the sole survivor in the absence of the predator population. When all population is present, the full system will be a classical food web. This model is similar to the model (Wolkowicz, 2006).

First we simplify the system (1) by choosing the dimensionless variables. Let:

$$
\begin{aligned}
& \bar{s}=\frac{s}{s^{0}}, \bar{x}_{i}=\frac{x_{i}}{\eta_{i} s^{0}}, i=1,2, \bar{y}=\frac{y}{\eta_{3} s^{0}}, \bar{t}=t D, \eta_{3}=\gamma \eta_{1} \\
& \bar{p}_{i}(\bar{s})=\frac{p_{i}\left(s^{0} \bar{s}\right)}{D}, i=1,2,3, \bar{q}\left(\bar{x}_{1}\right)=\frac{q\left(\bar{x}_{1} \eta_{1} s^{0}\right)}{D}
\end{aligned}
$$

Then dropping the bars, the system (1) becomes:

$$
\begin{aligned}
& s^{\prime}(t)=1-s(t)-\sum_{i=1}^{2} x_{i}(t) p_{i}(s(t))-y(t) p_{3}(s(t)) \\
& x_{1}{ }^{\prime}(t)=x_{1}(t)\left(-1+p_{1}(s(t))\right)-y(t) q\left(x_{1}(t)\right) \\
& x_{2}{ }^{\prime}(t)=x_{2}(t)\left(-1+p_{2}(s(t))\right) \\
& y^{\prime}(t)=y(t)\left(-1+p_{3}(s(t))+q\left(x_{1}(t)\right)\right) \\
& \text { with } s_{0} \geq 0, x_{i 0} \geq 0, i=1,2, y_{0} \geq 0 .
\end{aligned}
$$

The aforesaid assumptions hold good for system (2) also. Therefore, without loss of generality, one can consider the system (2) instead of the system (1) and can always reinterpret their findings in terms of original variables.

\section{Basic Properties}

\section{Boundedness and Dissipativity}

In this section, we shall prove that the solution trajectories of model (1) initiated in $\mathrm{R}_{+}^{4}$ are bounded, positively invariant and dissipative for all future time.

\section{Theorem 1}

Let the region $\Gamma$ be defined by:
$\Gamma=\left\{\left(s, x_{1}, x_{2}, y\right) \in \mathrm{R}_{+}^{4} \mid s+\sum_{i=1}^{2} x_{i}+y=1\right\}$

Then (i) $\Gamma$ is positively invariant, (ii) The solution trajectories of the system (2) initiated in $R_{+}^{4}$ are eventually bounded uniformly and enters into $\Gamma$ and (iii) System (2) is dissipative.

\section{Proof}

Let $s_{0}>0$ and consider that $s^{\prime}=1-s-\sum_{i=1}^{2} x_{i} p_{i}(s)-y p_{3}(s)$ $<1-s$.

Then:

$$
s(t)<1-\left(1-s_{0}\right) e^{-t} \text { for } s_{0}>0 \text { and } \limsup _{t \rightarrow \infty} s(t)<1
$$

For:

$$
x_{1}{ }^{\prime}=x_{1}\left(-1+p_{1}(s)\right)-y q\left(x_{1}\right)<x_{1}\left(-1+p_{1}(s)\right)<\delta_{1} x_{1}
$$

where:

$$
\delta_{1}=\left(-1+\max _{s \in \Gamma} p_{1}(s)\right)<0
$$

Then:

$$
x_{1}(t)<x_{10} e^{\delta_{1} t}, \delta_{1}<0 \text {, thus } \limsup _{t \rightarrow \infty} x_{1}(t)<x_{10} \text { for } x_{10}>0
$$

Similarly, we consider the equation:

$$
x_{2}{ }^{\prime}=x_{2}\left(-1+p_{2}(s)\right)<\delta_{2} x_{2}
$$

where:

$$
\delta_{2}=\left(-1+\max _{s \in \Gamma} p_{2}(s)\right)<0
$$

Then:

$$
x_{2}(t)<x_{20} e^{\delta_{2} t}, \delta_{2}<0 \text {, hence } \limsup _{t \rightarrow \infty} x_{2}(t)<x_{20} \text { for } x_{20}>0
$$

For the $y$ equation:

$$
y^{\prime}=y\left(-1+p_{3}(s)+q\left(x_{1}\right)\right)<\delta_{3} y
$$

where:

$$
\delta_{3}=\left(-1+\max _{s \in \Gamma} p_{3}(s)+\max _{x_{1} \in \Gamma} q\left(x_{1}\right)\right)<0
$$


Then:

$$
y(t)<y_{0} e^{\delta_{3} t}, \delta_{3}<0 \text {, hence } \lim _{t \rightarrow \infty} \sup y(t)<y_{0} \text { for } y_{0}>0
$$

Finally, we define the function $\Psi(t)=s(t)+x_{1}(t)+$ $x_{2}(t)+y(t)$ and taking time derivative along the trajectories of system (2) yields $\Psi^{\prime}(t)=1-\Psi(t)$ with $\Psi(0)$ $=s(0)+x_{1}(0)+x_{2}(0)+y(0) \geq 0$. Obviously $\Psi(t)=1+$ $\Psi(0) e^{-t}$ for $t \geq 0$ and gives that $\lim _{t \rightarrow \infty} \Psi(t)=1$.

Therefore, the trajectories of the system (2) are bounded uniformly and hence the system (2) is dissipative (El-Sheikh and Mahrouf, 2005). This completes the proof.

\section{Equilibria: Existence and their Local Stability}

The equilibrium points of the system (2) are the solutions of the following system of equations:

$$
\begin{aligned}
1-s-x_{1} p_{1}(s)-x_{2} p_{2}(s)-y p_{3}(s) & =0, \\
x_{1}\left(-1+p_{1}(s)\right)-y q\left(x_{1}\right) & =0, \\
x_{2}\left(-1+p_{2}(s)\right) & =0, \\
y\left(-1+p_{3}(s)+q\left(x_{1}\right)\right) & =0,
\end{aligned}
$$

with the assumed hypotheses of functions $p_{i}, q, i=1,2,3$. The eight possible equilibria of the form $E\left(s, x_{1}, x_{2}, y\right)$ are given as follows:

- Extinction of all populations: $E_{1}(1,0,0,0)$

- Survival of population $x_{1}$ only: $E_{2}\left(\lambda_{1}, 1-\lambda_{1}, 0,0\right)$

- Survival of population $x_{2}$ only: $E_{3}\left(\lambda_{2}, 0,1-\lambda_{2}, 0\right)$ where $\lambda_{i}, i=1,2$ are the unique solution of $p_{i}(s)-1=$ 0 (if exists)

- $\quad$ Survival of populations $x_{1}$ and $x_{2}$ only: $E_{4}\left(\breve{s}_{,} \breve{x}_{1}, \breve{x}_{2}, 0\right)$, where $\breve{s}$ is defined by the equations, $p_{1}(s)=p_{2}(s)=$ 1 with $\breve{s} \in(0,1)$ and $\breve{x}_{1}, \breve{x}_{2}$ are the solutions of $x_{1}+x_{2}=1-\breve{s}$

- Survival of population $y$ only: $E_{5}\left(\lambda_{3}, 0,0,1-\lambda_{3}\right)$, where $\lambda_{3}$ is defined as the unique solution of $p_{3}(s)-1$ $=0$ (if exists)

- $\quad$ Survival of population $x_{1}$ and $y$ only: $E_{6}\left(\tilde{s}, \tilde{x}_{1}, 0, \tilde{y}\right)$, where $\tilde{s}$ and $\tilde{x}_{1}$ are defined as the unique solutions of $p_{3}(s)+q\left(x_{1}\right)=1$ and they satisfy the equation:

$$
\tilde{s}+\tilde{x}_{1} p_{1}(\tilde{s})+p_{3}(\tilde{s}) \frac{\tilde{x}_{1}\left(-1+p_{1}(\tilde{s})\right)}{1-p_{3}(\tilde{s})}=1
$$

with $\tilde{s} \in(0,1)$ and $\tilde{y}=\frac{\tilde{x}_{1}\left(-1+p_{1}(\tilde{s})\right)}{1-p_{3}(\tilde{s})}$.

- $\quad$ Survival of population $x_{2}$ and $y$ only: $E_{7}\left(\hat{s}, 0, \hat{x}_{2}, \hat{y}\right)$, where $\hat{s}$ is defined by the equations, $p_{1}(s)=p_{2}(s)=$
1 with $\hat{s} \in(0,1)$ and $\hat{x}_{2}, \hat{y}$ are the solutions of $x_{2}+y=1-\hat{s}$

- Survival of all three populations: $E_{8}\left(\lambda_{2}, \bar{x}_{1}, \bar{x}_{2}, \bar{y}\right)$, where $\bar{x}_{1}$ is defined as the unique solution of

$$
\begin{aligned}
& q\left(x_{1}\right)=1-p_{3}\left(\lambda_{2}\right), \quad \bar{y}=\frac{\bar{x}_{1}\left(-1+p_{1}\left(\lambda_{2}\right)\right)}{q\left(\bar{x}_{1}\right)} \quad \text { and } \\
& \bar{x}_{2}=1-\lambda_{2}-\bar{x}_{1} p_{1}\left(\lambda_{2}\right)-\bar{y} p_{3}\left(\lambda_{2}\right)
\end{aligned}
$$

The values $\lambda_{i}$ represent the break-even concentration of nutrient.

Now we say that an equilibrium point will exist if its components are non-negative. The washout equilibrium point $E_{1}(1,0,0,0)$ always exists. Since $p_{i}$ is increasing with $p_{i}(0)=0, \lambda_{i}$ exist and satisfying:

$0<\lambda_{i}<1$ and $p_{i}\left(\lambda_{i}\right)=1 \Leftrightarrow p_{i}(1)>1$

In this case, there are equilibrium points $E_{2}, E_{3}$ and $E_{5}$ respectively, otherwise, no such equilibrium points exist. Now $E_{4}$ is a equilibrium point provided $\breve{x}_{1}>0, \breve{x}_{2}>0$ with $\breve{s} \in(0,1)$. Similar conditions hold for $E_{7}$. In case of the equilibrium point $E_{6}$, since $p_{3}, q$ are increasing with $p_{3}(0)=q(0)=0, \tilde{s}$ and $\tilde{x}_{1}$ exist and satisfy:

$p_{3}(\tilde{s})+q\left(\tilde{x}_{1}\right)=1$
$\Leftrightarrow p_{3}(1)+\lim _{x_{1} \rightarrow \infty} q\left(x_{1}\right)>1$

For the existence of $E_{6}$, we have two cases as in (El-Owaidy and Moniem, 2003):

Case 1: $1-p_{3}(\tilde{s})=q\left(\tilde{x}_{1}\right)>0$, then no condition is necessary

Case $2: \quad-1+p_{1}(\tilde{s})>0 \Rightarrow \tilde{s}>\lambda_{1}$

Note that $F(s)=1-s-\tilde{x}_{1} p_{1}(s)-p_{3}(s) \frac{\tilde{x}_{1}\left(-1+p_{1}(s)\right)}{1-p_{3}(s)}$ is decreasing in $s$ with $F(0)=1, F(\tilde{s})=0 \quad$ and $F\left(\lambda_{1}\right)=1-\lambda_{1}-\tilde{x}_{1}$. So $\tilde{s}>\lambda_{1}$ if and only if $\tilde{x}_{1}<1-\lambda_{1}$. Therefore, $E_{6}$ exists if and only if:

$\lambda_{1}<1$ and $\tilde{x}_{1}<1-\lambda_{1}$

Finally, the feasibility conditions for the mixed culture (interior) equilibrium point $E_{8}$ are $\bar{x}_{1}>0, \bar{x}_{2}>0, \bar{y}>0$. In order for $\bar{x}_{1}>0$, we require $\lambda_{3}>\lambda_{2}$. Similar calculations show that $\bar{y}>0 \Rightarrow \lambda_{2}>\lambda_{1}$ and $\bar{x}_{2}>0 \Rightarrow \lambda_{2}<1$. Therefore, $E_{8}$ exists if and only if:

$\lambda_{3}>\lambda_{2}>\lambda_{1}>0$ and $\lambda_{2}<1$ 
Next, the local stability analysis of the eight equilibrium points will be investigated by finding the eigenvalues of the associated Jacobian matrices. The Jacobian matrix due to the linearization of (2) about an arbitrary equilibrium $E\left(s, x_{1}, x_{2}, y\right) \in \mathbb{R}_{+}^{4}$ is given by:

$$
J(E)=\left[J_{i j}\right]_{4 \times 4}
$$

where:

$$
\begin{aligned}
& J_{11}=-1-x_{1} p_{1}{ }^{\prime}(s)-x_{2} p_{2}{ }^{\prime}(s)-y p_{3}{ }^{\prime}(s), \\
& J_{12}=-p_{1}(s), J_{13}=-p_{2}(s), J_{14}=-p_{3}(s), \\
& J_{21}=x_{1} p_{1}{ }^{\prime}(s), J_{22}=-1+p_{1}(s)-y q^{\prime}\left(x_{1}\right), J_{23}=0, \\
& J_{24}=-q\left(x_{1}\right), J_{31}=x_{2} p_{2}{ }^{\prime}(s), J_{32}=0, J_{33}=-1+p_{2}(s), \\
& J_{34}=0, J_{41}=y p_{3}{ }^{\prime}(s), J_{42}=y q^{\prime}\left(x_{1}\right), J_{43}=0, \\
& J_{44}=-1+p_{3}(s)+q\left(x_{1}\right) .
\end{aligned}
$$

The dynamic behavior of plane equilibria $E_{1}, E_{2}, E_{3}$, $E_{4}, E_{5}$ and $E_{7}$ have been stated in the form of Theorems [2-7] respectively. We left the proofs as these directly follow from the Routh-Hurwitz criteria.

\section{Theorem 2}

If $\lambda_{i}>1, i=1,2,3$, then the washout equilibrium point $E_{1}$ is Locally Asymptotically Stable (LAS) that is, hyperbolic sink and hyperbolic saddle if otherwise.

\section{Theorem 3}

Suppose $\lambda_{1}<1$. (i) If $1-\tilde{x}_{1}<\lambda_{1}<\lambda_{2}$, then $E_{2}$ is LAS (hyperbolic sink). (ii) If $1-\tilde{x}_{1}>\lambda_{1}>\lambda_{2}$, then $E_{2}$ is hyperbolic saddle and is repelling locally in both directions of $x_{2}$ and $y$. In particular, the stable manifold $W^{+}$and the unstable manifold $W$ both are of dimensions two and given by $\operatorname{Dim}^{+}\left(E_{2}\left(\lambda_{1}, 1-\lambda_{1}, 0,0\right)\right)=2$, $\operatorname{DimW}^{-}\left(E_{2}\left(\lambda_{1}, 1-\lambda_{1}, 0,0\right)\right)=2$.

\section{Theorem 4}

Suppose $\lambda_{2}<1$. (i) If $\lambda_{2}<\min \left\{\lambda_{1}, \lambda_{3}\right\}$, then the equilibrium point $E_{3}$ is hyperbolic sink. (ii) If $\lambda_{2}>\max \left\{\lambda_{1}, \lambda_{3}\right\}$, then $E_{3}$ is a hyperbolic saddle and is repelling locally in both directions of $x_{1}$ and $y$. In particular, $\operatorname{Dim} W^{+}\left(E_{3}\right)=2, \operatorname{Dim}^{-}\left(E_{3}\right)=2$.

\section{Theorem 5}

Suppose $\lambda_{3}<1$. (i) If $\lambda_{3}<\lambda_{2}$ and $p_{1}\left(\lambda_{3}\right)-\left(1-\lambda_{3}\right) q^{\prime}(0)<1$, then the equilibrium point $E_{5}$ is hyperbolic sink. (ii) If the inequalities in (i) are reversed, then $E_{5}$ is a hyperbolic saddle point and is repelling locally in both directions of $x_{1}$ and $x_{2}$.

\section{Theorem 6}

Suppose $E_{4}$ exists. Let $\breve{a}_{1}>0, \breve{a}_{2}>0$ and $\breve{a}_{1} \breve{a}_{2}>\breve{a}_{3}$. (i) If $p_{3}(\breve{s})+q\left(\breve{x}_{1}\right)<1$, then $E_{4}$ is hyperbolic sink. (ii) If $p_{3}(\breve{s})+q\left(\breve{x}_{1}\right)>1$, then $E_{4}$ is a hyperbolic saddle point and is repelling locally in direction of $y$.

\section{Theorem 7}

Suppose $E_{7}$ exists. Let $\hat{a}_{1}>0, \hat{a}_{2}>0, \hat{a}_{1} \hat{a}_{2}>\hat{a}_{3}$. (i) If $p_{1}(\hat{s})-\hat{y} q^{\prime}(0)<1$, then $E_{7}$ is hyperbolic sink. (ii) If $p_{1}(\hat{s})-\hat{y} q^{\prime}(0)>1$, then $E_{7}$ is a hyperbolic saddle point and is repelling locally in direction of $x_{1}$.

Next, we perform the linearized stability analyses for the rest point $E_{6}$. The Jacobian matrix due to linearization of system (2) around $E_{6}$ is given by:

$$
J\left(E_{6}\right)=\left[\begin{array}{cccc}
m_{11} & m_{12} & m_{13} & m_{14} \\
m_{21} & m_{22} & 0 & m_{24} \\
0 & 0 & m_{33} & 0 \\
m_{41} & m_{42} & 0 & 0
\end{array}\right]
$$

where:

$$
\begin{aligned}
& m_{11}=-1-\tilde{x}_{1} p_{1}{ }^{\prime}(\tilde{s})-\tilde{y} p_{3}{ }^{\prime}(\tilde{s}), m_{12}=-p_{1}(\tilde{s}), \\
& m_{13}=-p_{2}(\tilde{s}), m_{14}=-p_{3}(\tilde{s}), m_{21}=\tilde{x}_{1} p_{1}{ }^{\prime}(\tilde{s}), \\
& m_{22}=-1+p_{1}(\tilde{s})-\tilde{y} q^{\prime}\left(\tilde{x}_{1}\right), m_{24}=-q\left(\tilde{x}_{1}\right), \\
& m_{33}=-1+p_{2}(\tilde{s}), m_{41}=\tilde{y} p_{3}(\tilde{s}), \\
& m_{42}=\tilde{y} q^{\prime}\left(\tilde{x}_{1}\right)
\end{aligned}
$$

The eigenvalues of the matrix $J\left(E_{6}\right)$ are given by:

$$
r_{1}=m_{33}=-1+p_{2}(\tilde{s})
$$

and $r_{2}, r_{3}, r_{4}$ satisfy the equation:

$$
r^{3}+\tilde{a}_{1} r^{2}+\tilde{a}_{2} r+\tilde{a}_{3}=0
$$

where:

$$
\begin{aligned}
& \tilde{a}_{1}=-m_{11}-m_{22}, \\
& \tilde{a}_{2}=-m_{24} m_{42}+m_{11} m_{22}-m_{12} m_{21}-m_{14} m_{41}, \\
& \tilde{a}_{3}=m_{11} m_{24} m_{42}-m_{12} m_{24} m_{41}-m_{14} m_{21} m_{42}+m_{14} m_{22} m_{41} .
\end{aligned}
$$

We summarize above discussion as follows.

\section{Theorem 8}

Suppose (8) holds. (i) If $\tilde{s}>\lambda_{2}$ and $\tilde{a}_{1}>0, \tilde{a}_{3}>0$, $\tilde{a}_{1} \tilde{a}_{2}>\tilde{a}_{3}$, then $E_{6}$ is a hyperbolic saddle and is repelling locally in direction of $x_{2}$. (ii) The equilibrium point $E_{6}$ is hyperbolic sink, if $\tilde{s}<\lambda_{2}$ and $\tilde{a}_{1}>0, \tilde{a}_{3}>0, \tilde{a}_{1} \tilde{a}_{2}>\tilde{a}_{3}$ hold concurrently. In particular, the stable manifold 
$W^{+}\left(E_{6}=\left(\tilde{s}, \tilde{x}_{1}, 0, \tilde{y}\right)\right)$ is the $s-x_{1}-y$ space and the unstable manifold $W^{-}\left(E_{6}\right)$ is the $x_{2}$ direction, that is $\operatorname{Dim}^{+}\left(E_{6}\right)=3, \operatorname{Dim}^{-}\left(E_{6}\right)=1$.

Finally, when the interior equilibrium point $E_{8}\left(\bar{s}, \bar{x}_{1}, \bar{x}_{2}, \bar{y}\right)$ exists, the Jacobian matrix at $E_{8}$ is given by:

$J\left(E_{8}\right)=\left[\begin{array}{cccc}n_{11} & n_{12} & n_{13} & n_{14} \\ n_{21} & n_{22} & 0 & n_{24} \\ n_{31} & 0 & 0 & 0 \\ n_{41} & n_{42} & 0 & 0\end{array}\right]$

where:

$$
\begin{aligned}
& n_{11}=-1-\bar{x}_{1} p_{1}{ }^{\prime}\left(\lambda_{2}\right)-\bar{x}_{2} p_{2}{ }^{\prime}\left(\lambda_{2}\right)-\bar{y} p_{3}{ }^{\prime}\left(\lambda_{2}\right), \\
& n_{12}=-p_{1}\left(\lambda_{2}\right), n_{13}=-1, n_{14}=-p_{3}\left(\lambda_{2}\right), \\
& n_{21}=\bar{x}_{1} p_{1}{ }^{\prime}\left(\lambda_{2}\right), n_{22}=-1+p_{1}\left(\lambda_{2}\right)-\bar{y} q^{\prime}\left(\bar{x}_{1}\right), \\
& n_{24}=-q\left(\bar{x}_{1}\right), n_{31}=\bar{x}_{2} p_{2}{ }^{\prime}\left(\lambda_{2}\right), n_{41}=\bar{y} p_{3}{ }^{\prime}\left(\lambda_{2}\right), \\
& n_{42}=\bar{y} q^{\prime}\left(\bar{x}_{1}\right)
\end{aligned}
$$

The eigenvalues of $J\left(E_{8}\right)$ satisfy the equation:

$$
r^{4}+\bar{a}_{1} r^{3}+\bar{a}_{2} r^{2}+\bar{a}_{3} r+\bar{a}_{4}=0
$$

where:

$$
\begin{aligned}
& \bar{a}_{1}=-\operatorname{Trace} J\left(E_{8}\right)=-n_{11}-n_{22} \\
& \bar{a}_{2}=-n_{12} n_{21}-n_{24} n_{42}+n_{11} n_{22}-n_{14} n_{41}+n_{31} \\
& \bar{a}_{3}=-n_{22} n_{31}+n_{11} n_{24} n_{42}-n_{12} n_{24} n_{41}-n_{14} n_{21} n_{42}+n_{14} n_{22} n_{41} \\
& \bar{a}_{4}=\operatorname{det} J\left(E_{8}\right)=-n_{24} n_{31} n_{42}
\end{aligned}
$$

Therefore by Routh-Hurwitz criteria $E_{8}$ will be LAS if and only if $\bar{a}_{1}>0, \bar{a}_{2}>0, \bar{a}_{3}>0, \bar{a}_{4}>0, \bar{a}_{1} \bar{a}_{2}>\bar{a}_{3}$ and $\bar{a}_{1} \bar{a}_{2} \bar{a}_{3}>\bar{a}_{3}^{2}+\bar{a}_{4} \bar{a}_{1}^{2}$.

Thus we summarize above result in the following theorem.

\section{Theorem 9}

Suppose (9) holds. Then $E_{8}$ is LAS if $\bar{a}_{1}>0, \bar{a}_{2}>0, \bar{a}_{3}>0, \bar{a}_{4}>0, \quad \bar{a}_{1} \bar{a}_{2}>\bar{a}_{3}$ $\bar{a}_{1} \bar{a}_{2} \bar{a}_{3}>\bar{a}_{3}^{2}+\bar{a}_{4} \bar{a}_{1}^{2}$, otherwise unstable.

\section{Global Stability Analysis}

In the last section, we showed the existence of all equilibria and their local stability. Here, we shall present the global stability analysis of system (2) around the equilibria.

\section{Theorem 10}

If $\lambda_{i}>1$, then the solution trajectories of the system (2) satisfy $\lim _{t \rightarrow+\infty}\left(s(t), x_{1}(t), x_{2}(t), y(t)\right)=(1,0,0,0)$.

\section{Proof}

The proof is very straightforward and similar to (Li and Kuang, 2000; Nasrin and Rana, 2011).

Now we show that the system (2) is persistent by showing that a positive equilibrium point of any 3dimensional subspace of $\mathrm{R}_{s x_{1} x_{2} y}^{+}$, is globally asymptotically stable with respect to trajectories originating from the interior of it. First we establish criterion for the global stability of $E_{2}\left(\lambda_{1}, 1-\lambda_{1}, 0,0\right)$.

Consider system (2) restricted to $\mathrm{R}_{s x_{1}}^{+}$:

$s^{\prime}(t)=1-s-x_{1} p_{1}(s)$
$x_{1}{ }^{\prime}(t)=x_{1}\left(-1+p_{1}(s)\right)$

We have shown that $\bar{E}_{2}\left(\lambda_{1}, 1-\lambda_{1}\right)$ and consequently $E_{2}\left(\lambda_{1}, 1-\lambda_{1}, 0,0\right)$ exists if $\lambda_{1}<1$. For any point in $\mathrm{R}_{s x_{1}}^{+}$, let $G$ be a neighborhood of it. In $\mathrm{R}_{s x_{1}}^{+}$, we choose the Liapunov function $V$ such that:

$V\left(s, x_{1}\right)=\frac{1}{2} c_{1}(s-\widehat{s})^{2}+\frac{1}{2} c_{2}\left(x_{1}-\widehat{x}_{1}\right)^{2}$

where, $c_{1}, c_{2} \in \mathrm{R}_{+}$and $\hat{s}=\lambda_{1}, \widehat{x}_{1}=1-\lambda_{1}$ are solutions of (17). Then $\mathrm{V}$ is a positive definite in $\mathrm{R}_{s x_{1}}^{+}$and also a Liapunov function for (17) in $G$.

Now:

$V^{\prime}=c_{1}(s-\widehat{s})\left(1-s-x_{1} p_{1}(s)\right)+c_{2}\left(x_{1}-\widehat{x}_{1}\right) x_{1}\left(-1+p_{1}(s)\right)$

along the trajectories of (17) in $\mathrm{R}_{s x_{1}}^{+}$.

But from (17), we have $1-\widehat{s}-\widehat{x}_{1} p_{1}(\widehat{s})=0$, $-1+p_{1}(\widehat{s})=0$.

Thus:

$$
\begin{aligned}
& V^{\prime}=c_{1}(s-\widehat{s})\left(\widehat{s}+\widehat{x}_{1} p_{1}(\widehat{s})-s-x_{1} p_{1}(s)\right) \\
& +c_{2}\left(x_{1}-\widehat{x}_{1}\right) x_{1}\left(-p_{1}(\widehat{s})+p_{1}(s)\right) \\
& =-c_{1}(s-\widehat{s})^{2}+c_{1}(s-\widehat{s})\left(\widehat{x}_{1} p_{1}(\widehat{s})-x_{1} p_{1}(s)\right) \\
& +c_{2}\left(x_{1}-\widehat{x}_{1}\right) x_{1}\left(p_{1}(s)-p_{1}(\widehat{s})\right) \\
& =c_{11}(s-\widehat{s})^{2}+\frac{1}{2} c_{12}(s-\widehat{s})\left(x_{1}-\widehat{x}_{1}\right) \\
& +\frac{1}{2} c_{21}(s-\widehat{s})\left(x_{1}-\widehat{x}_{1}\right)+c_{22}\left(x_{1}-\widehat{x}_{1}\right)^{2}
\end{aligned}
$$

where:

$$
\begin{aligned}
& c_{11}=-c_{1}<0, c_{12}=c_{21}=c_{1} \frac{\left(\widehat{x}_{1} p_{1}(\widehat{s})-x_{1} p_{1}(s)\right)}{\left(x_{1}-\widehat{x}_{1}\right)}, \\
& c_{22}=\frac{x_{1}\left(p_{1}(s)-p_{1}(\widehat{s})\right)}{\left(x_{1}-\widehat{x}_{1}\right)}
\end{aligned}
$$


In particular:

$V^{\prime}=X^{t} C X=\langle C X, X\rangle$

where:

$$
X=\left(\begin{array}{l}
v_{1} \\
v_{2}
\end{array}\right)=\left(\begin{array}{c}
s-\widehat{s} \\
x_{1}-\widehat{x}_{1}
\end{array}\right) \text { and } C=\left(\begin{array}{cc}
c_{11} & \frac{1}{2} c_{12} \\
\frac{1}{2} c_{12} & c_{22}
\end{array}\right)
$$

It is clear that $C$ is a real symmetric matrix and $C=\frac{1}{2}\left(C^{t}+C\right)$ where $t$ stands for transpose.

Let $\Gamma_{0}=\left\{\left(s, x_{1}\right) \in \mathrm{R}_{s x_{1}}^{+} \mid s>0, x_{1}>0, s+x_{1}=1\right\}$.

Define two sets as:

$$
\begin{aligned}
& S_{1}=\left\{\left(s, x_{1}\right) \in \Gamma_{0} \cap \operatorname{int} \mathrm{R}_{s x_{1}}^{+} \mid V\left(s, x_{1}\right)=0\right\}, \\
& S_{2}=\left\{\left(s, x_{1}\right) \in \operatorname{int} \mathrm{R}_{s x_{1}}^{+} \mid s=\widehat{s}, x_{1}=\widehat{x}_{1}\right\}
\end{aligned}
$$

Then it is obvious that the sets are identical, i.e., $S_{1} \equiv S_{2}$.

Next we define the set $E$ as $\mathrm{E}=\left\{\left(s, x_{1}\right) \in \mathrm{R}_{s x_{1}}^{+} \mid V^{\prime}=0\right\} \cap \bar{G}$.

Then $E_{2}\left(\lambda_{1}, 1-\lambda_{1}, 0,0\right)$ is the largest invariant set in $\mathrm{E}$ restricted to $\mathrm{R}_{s x_{1}}^{+}$.

Hence we conclude from LaSalle's Invariant Principle (LaSalle and Lefchetz, 1961) that $\bar{E}_{2}\left(\lambda_{1}, 1-\lambda_{1}\right)$ or consequently $E_{2}\left(\lambda_{1}, 1-\lambda_{1}, 0,0\right)$ is globally asymptotically stable with respect to trajectories originating from int $R_{s x_{1}}^{+}$ if the matrix $C$ is a negative definite.

\section{Theorem 11}

The equilibrium point $E_{2}\left(\lambda_{1}, 1-\lambda_{1}, 0,0\right)$ in $\mathrm{R}_{s x_{1}}^{+}$is globally asymptotically stable with respect to solution trajectories originating from int $\mathrm{R}_{s \gamma_{1}}^{+}$if:

$$
\text { (i) } c_{22}<0(i i) c_{11} c_{22}-\frac{1}{4} c^{2}{ }_{12}>0 \text {. }
$$

\section{Proof}

The proof is very straightforward and similar to (Nani and Freedman, 2000).

Now, we shall deduce the global asymptotic stability criteria of $E_{6}\left(\tilde{s}, \tilde{x}_{1}, 0, \tilde{y}\right)$ with respect to solution trajectories initiating from int $\mathrm{R}_{s x, y}^{+}$.

For the system (2) restricted in $\mathrm{R}_{s x, y}^{+}$, we choose the Liapunov function:

$$
W\left(s, x_{1}, y\right)=s-\tilde{s}-\tilde{s} \ln \left(\frac{s}{\tilde{s}}\right)+\frac{1}{2} k_{1}\left(x_{1}-\tilde{x}_{1}\right)^{2}+\frac{1}{2} k_{2}(y-\tilde{y})^{2}
$$

where, $k_{i} \in \mathrm{R}_{+}$for $i=1,2$.

Then:

$$
\begin{aligned}
& W^{\prime}=\left(\frac{s-\tilde{s}}{s}\right)\left(1-s-x_{1} p_{1}(s)-y p_{3}(s)\right) \\
& +k_{1}\left(x_{1}-\tilde{x}_{1}\right)\left(x_{1}\left(-1+p_{1}(s)\right)-y q\left(x_{1}\right)\right) \\
& +k_{2}(y-\tilde{y}) y\left(-1+p_{3}(s)+q\left(x_{1}\right)\right)
\end{aligned}
$$

along the trajectories of the system (2) restricted in $\mathbb{R}_{s i, y}^{+}$.

But we have $\quad 1-\tilde{s}-\tilde{x}_{1} p_{1}(\tilde{s})-\tilde{y} p_{3}(\tilde{s})=0$, $\tilde{x}_{1}\left(-1+p_{1}(\tilde{s})\right)-\tilde{y} q\left(\tilde{x}_{1}\right)=0,-1+p_{3}(\tilde{s})+q\left(\tilde{x}_{1}\right)=0$.

Thus (23) reduces to:

$$
\begin{aligned}
& W^{\prime}=\left(\frac{s-\tilde{s}}{s}\right)\left(\tilde{s}+\tilde{x}_{1} p_{1}(\tilde{s})+\tilde{y} p_{3}(\tilde{s})-s-x_{1} p_{1}(s)-y p_{3}(s)\right) \\
& \left.+k_{1}\left(x_{1}-\tilde{x}_{1}\right)\left(x_{1}\left(-p_{1}(\tilde{s})\right)+\frac{\tilde{y} q\left(\tilde{x}_{1}\right)}{\tilde{x}_{1}}+p_{1}(s)\right)-y q\left(x_{1}\right)\right) \\
& +k_{2}(y-\tilde{y}) y\left(-p_{3}(\tilde{s})-q\left(\tilde{x}_{1}\right)+p_{3}(s)+q\left(x_{1}\right)\right) . \\
& =-\frac{(s-\tilde{s})^{2}}{s}+\left\{\frac{\tilde{x}_{1} p_{1}(\tilde{s})-x_{1} p_{1}(s)}{s\left(x_{1}-\tilde{x}_{1}\right)}\right\}(s-\tilde{s})\left(x_{1}-\tilde{x}_{1}\right) \\
& +\left\{\frac{\tilde{y} p_{3}(\tilde{s})-y p_{3}(s)}{s(y-\tilde{y})}\right\}(s-\tilde{s})\left(y-\tilde{y}_{1}\right) \\
& +\left\{-k_{1} \frac{\left.x_{1}\left(p_{1}(\tilde{s})\right)-p_{1}(s)\right)}{(s-\tilde{s})}\right\}(s-\tilde{s})\left(x_{1}-\tilde{x}_{1}\right) \\
& +\left\{k_{1} \frac{\tilde{y} q\left(\tilde{x}_{1}\right)}{\tilde{x}_{1}}-y q\left(x_{1}\right)\right\}\left(x_{1}-\tilde{x}_{1}\right)^{2} \\
& +\left\{-k_{2} y \frac{p_{3}(\tilde{s})-p_{3}(s)}{(s-\tilde{s})}\right\}(s-\tilde{s})(y-\tilde{y}) \\
& +\left\{-k_{2} y \frac{q\left(\tilde{x}_{1}\right)-q\left(x_{1}\right)}{(y-\tilde{y})}\right\}(y-\tilde{y})^{2}
\end{aligned}
$$

Let $X=\left(\begin{array}{c}v_{1} \\ v_{2} \\ v_{3}\end{array}\right)$ such that $\begin{aligned} v_{1} & =s-\tilde{s} \\ v_{2} & =x_{1}-\tilde{x}_{1} \\ v_{3} & =y-\tilde{y}\end{aligned}$ and set:

$$
\begin{aligned}
& b_{11}=-\frac{1}{s}<0, b_{12}=b_{21}=\frac{\tilde{x}_{1} p_{1}(\tilde{s})-x_{1} p_{1}(s)}{s\left(x_{1}-\tilde{x}_{1}\right)}-k_{1} \frac{\left.x_{1}\left(p_{1}(\tilde{s})\right)-p_{1}(s)\right)}{(s-\tilde{s})}, \\
& b_{22}=k_{1} \frac{x_{1} \frac{\tilde{y} q\left(\tilde{x}_{1}\right)}{\tilde{x}_{1}}-y q\left(x_{1}\right)}{\left(x_{1}-\tilde{x}_{1}\right)}, \\
& b_{13}=b_{31}=\frac{\tilde{y} p_{3}(\tilde{s})-y p_{3}(s)}{s(y-\tilde{y})}-k_{2} y \frac{p_{3}(\tilde{s})-p_{3}(s)}{(s-\tilde{s})}, \\
& b_{33}=-k_{2} y \frac{q\left(\tilde{x}_{1}\right)-q\left(x_{1}\right)}{(y-\tilde{y})}, b_{23}=b_{32}=0
\end{aligned}
$$


Then:

$V^{\prime}=b_{11} v_{1}^{2}+b_{22} v_{2}^{2}+b_{33} v_{3}^{2}+b_{12} v_{1} v_{2}+b_{13} v_{1} v_{3}$

where, $b_{i j}=b_{j i}$ with $b_{23}=b_{32}=0$. Therefore, we write:

$V^{\prime}=X^{t} B X=\langle B X, X\rangle$

where:

$$
B=\left(\begin{array}{ccc}
b_{11} & \frac{1}{2} b_{12} & \frac{1}{2} b_{13} \\
\frac{1}{2} b_{12} & b_{22} & \frac{1}{2} b_{23} \\
\frac{1}{2} b_{13} & \frac{1}{2} b_{23} & b_{33}
\end{array}\right)
$$

It is clear that $B$ is a real symmetric matrix such that $B=\frac{1}{2}\left(B^{t}+B\right)$ where $t$ stands for transpose. The eigenvalues of $B$ satisfy the equation:

$r^{3}+m_{1} r^{2}+m_{2} r+m_{3}=0$

where, $m_{1}=-$ trace $B=-\left(b_{11}+b_{22}+b_{33}\right)$ :

$$
\begin{aligned}
& m_{2}=\operatorname{det}\left|\begin{array}{cc}
b_{22} & \frac{1}{2} b_{23} \\
\frac{1}{2} b_{23} & b_{33}
\end{array}\right|+\operatorname{det}\left|\begin{array}{cc}
b_{11} & \frac{1}{2} b_{13} \\
\frac{1}{2} b_{13} & b_{33}
\end{array}\right|+\operatorname{det}\left|\begin{array}{cc}
b_{11} & \frac{1}{2} b_{12} \\
\frac{1}{2} b_{12} & b_{22}
\end{array}\right|, \\
& m_{3}=-\operatorname{det}(B)
\end{aligned}
$$

These reduce with $b_{23}=b_{32}=0$ to:

$$
\begin{aligned}
& m_{1}=-\left(b_{11}+b_{22}+b_{33}\right), \\
& m_{2}=b_{22} b_{33}+\left(b_{11} b_{33}-\frac{1}{4} b_{13}^{2}\right)+\left(b_{11} b_{22}-\frac{1}{4} b_{12}^{2}\right), \\
& m_{3}=\frac{1}{4} b_{13}^{2} b_{22}-b_{33}\left(b_{11} b_{22}-\frac{1}{4} b_{12}^{2}\right)
\end{aligned}
$$

Thus $B$ is negative definite if:

$$
m_{1}>0, m_{3}>0, m_{1} m_{2}>m_{3}
$$

Follows from the Routh-Hurwitz criteria and Lemma 6.1 of (Nani and Freedman, 2000).

Using Lemma 6.2 (Nani and Freedman, 2000), the criteria (28) gives the following result.

\section{Theorem 12}

The equilibrium point $\bar{E}_{6}\left(\tilde{s}, \tilde{x}_{1}, \tilde{y}\right) \in \mathrm{R}_{s x_{1} y}^{+}$is globally asymptotically stable if: (i) $b_{11}<0, b_{22}<0, b_{33}<0$

(ii) $b_{11} b_{33}-\frac{1}{4} b_{13}^{2}>0$

(iii) $b_{33}\left(b_{11} b_{22}-\frac{1}{4} b^{2}{ }_{12}\right)<\frac{1}{4} b^{2}{ }_{13} b_{22}$ with $b_{11} b_{22}-\frac{1}{4} b_{12}^{2}>0$

\section{Proof}

We left the proof as it is very straightforward and similar to (Nani and Freedman, 2000).

\section{Hopf-Andronov-Poincare Bifurcation}

In this section, we shall show that the system (2) undergoes a Hopf-Andronov-Poincare bifurcation by using $\mu$ as a bifurcation real parameter. Without loss of generality, suppose that $p_{1}$ is a function of $s$ and $\mu$. Then system (2) becomes:

$$
\begin{aligned}
& s^{\prime}(t)=1-s-x_{1} p_{1}(s ; \mu)-x_{2} p_{2}(s)-y p_{3}(s) \\
& x_{1}{ }^{\prime}(t)=x_{1}\left(-1+p_{1}(s ; \mu)\right)-y q\left(x_{1}\right) \\
& x_{2}{ }^{\prime}(t)=x_{2}\left(-1+p_{2}(s)\right) \\
& y^{\prime}(t)=y\left(-1+p_{3}(s)+q\left(x_{1}\right)\right) \\
& \text { with } s_{0} \geq 0, x_{i 0} \geq 0, i=1,2, y_{0} \geq 0 .
\end{aligned}
$$

Now system (29) can be written as:

$$
x^{\prime}(t)=F(x ; \mu), x(0)=x_{0}
$$

where, $\quad x=\left(s, x_{1}, x_{2}, y\right)^{t} \in \mathrm{R}^{4}, \quad x_{0}=\left(s_{0}, x_{10}, x_{20}, y_{0}\right)^{t} \quad$ and $\mu \in \mathrm{R}$ is the bifurcation real parameter. The function $F(x ; \mu)$ is a $C^{r}(r \geq 5)$ on an open set in $\mathrm{R}^{4} \times \mathrm{R}$.

Let:

$$
P_{\mu}=\left\{\begin{array}{l}
E_{2}\left(\lambda_{1}, 1-\lambda_{1}, 0,0 ; \mu\right), E_{3}\left(\lambda_{2}, 0,1-\lambda_{2}, 0 ; \mu\right), \\
E_{4}\left(\breve{s}_{x_{1}}, \breve{x}_{2}, 0 ; \mu\right), E_{5}\left(\lambda_{3}, 0,0,1-\lambda_{3} ; \mu\right), \\
E_{6}\left(\tilde{s}, \tilde{x}_{1}, 0, \tilde{y} ; \mu\right), E_{7}\left(\hat{s}, 0, \hat{x}_{2}, \hat{y} ; \mu\right), \\
E_{8}\left(\lambda_{2}, \bar{x}_{1}, \bar{x}_{2}, \bar{y} ; \mu\right)
\end{array}\right\}
$$

be the set of equilibria of system (29). Suppose that $G$ is a sufficiently large open set containing $E_{i} \in P_{\mu}$ such that $F\left(P_{\mu}\right)=0$ for $\mu \in \mathrm{R}$. We are now interested to vary $\mu$ in order to observe the qualitative changes of solution trajectories near $P_{\mu}$.

\section{Corollary 1}

Assume that Theorems [3-7] are hold. Then there is no Hopf bifurcation at $E_{2}, E_{3}, E_{4}, E_{5}$ and $E_{7}$.

\section{Proof}

If Theorems [3-7] hold respectively, then $E_{2}, E_{3}, E_{4}$, $E_{5}$ and $E_{7}$ are repeller and their stable manifolds lie along an axis. 
Next we need to compute stability of $E_{6}\left(\tilde{s}, \tilde{x}_{1}, 0, \tilde{y} ; \mu\right)$ and $E_{8}\left(\lambda_{2}, \bar{x}_{1}, \bar{x}_{2}, \bar{y} ; \mu\right)$ and then vary $\mu$ so as to obtain a desired Hopf bifurcation at $\mu=\mu_{1}^{*}$ and $\mu=\mu_{2}^{*}$ around $E_{6}$ and $E_{8}$ respectively.

Firstly, Hopf bifurcation analysis for $E_{6}\left(\tilde{s}, \tilde{x}_{1}, 0, \tilde{y} ; \mu\right)$ :

The linearization of system (29) about $E_{6}\left(\tilde{s}, \tilde{x}_{1}, 0, \tilde{y} ; \mu\right)$ for any values of $\mu$ is given by:

$$
\zeta^{\prime}=J_{\mu}\left(E_{6}\right) \zeta, \zeta \in \mathrm{R}^{4}
$$

where, $J_{\mu}\left(E_{6}\right)$ is defined as in (11) and its nonzero elements are defined as in (12), with $p_{1}(\tilde{s})$ and $p_{1}^{\prime}(\tilde{s})$ replaced by $p_{1}(\tilde{s} ; \mu) \& p_{1}{ }^{\prime}(\tilde{s} ; \mu)$ respectively.

The eigenvalues of the matrix $J_{\mu}\left(E_{6}\right)$ are $r_{1}=m_{33}=-1+p_{2}(\tilde{s})$ and $r_{i}, i=2,3,4$ satisfy the equation

$$
r^{3}+b_{1} r^{2}+b_{2} r+b_{3}=0
$$

where, $b_{i}=\tilde{a}_{i}, i=1,2,3$ are defined as in (13).

By the Routh-Hurwitz criteria, all the roots of (31) will have negative real parts if:

$$
R_{1}: b_{1}>0, b_{3}>0 \text { and } R_{2}: b_{1} b_{2}>b_{3}
$$

It is required for a Hopf bifurcation that either $R_{1}$ or $R_{1}$ must be violated. Suppose that $b_{1}>0, b_{3}>0$, then clearly (31) has two purely imaginary roots if and only if

$$
b_{1} b_{2}=b_{3}
$$

for some values of $\mu$, say $\mu_{1}^{*}$. Since $b_{2}>0$ at $\mu=\mu_{1}^{*}$, there is an open interval containing $\mu_{1}^{*}$, say $\left(\mu_{1}^{*}-\varepsilon, \mu_{1}^{*}+\varepsilon\right)$ for some $\varepsilon>0$ for which $\mu_{1}^{*}-\varepsilon>0$, such that $b_{2}>0$ for $\mu \in\left(\mu_{1}^{*}-\varepsilon, \mu_{1}^{*}+\varepsilon\right)$. Thus for $\mu \in\left(\mu_{1}^{*}-\varepsilon, \mu_{1}^{*}+\varepsilon\right)$, the characteristic equation (31) cannot have positive real roots. For $\mu=\mu_{1}^{*}$, we have (Freedman and Ruan, 1992, p.80):

$$
\left(r^{2}+b_{2}\right)\left(r+b_{1}\right)=0
$$

The roots of (33) are $r_{2}=i \sqrt{b_{2}}, r_{3}=-i \sqrt{b_{2}}, r_{4}=-b_{1}$.

For $\mu \in\left(\mu_{1}^{*}-\varepsilon, \mu_{1}^{*}+\varepsilon\right)$, we write the roots in the general form:

$$
\begin{aligned}
& r_{2}(\mu)=\alpha(\mu)+i \beta(\mu), r_{3}(\mu)=\alpha(\mu)-i \beta(\mu) \\
& \text { and } r_{4}(\mu)=-b_{1}(\mu)
\end{aligned}
$$

For the application of Hopf's bifurcation theory to the system (29) (Marsden and Mckracken, 1976), it is required to satisfy the transversality condition:
$\operatorname{Re}\left[\frac{d r_{i}}{d \mu}\right]_{\mu=\mu_{i}^{*}} \neq 0, i=2,3$

Substituting $r_{2}(\mu)=\alpha(\mu)+i \beta(\mu)$ and $r_{3}(\mu)=\alpha(\mu)+$ $i \beta(\mu)$ into (31) and calculating the derivatives with respect to $\mu$, we obtain:

$$
\begin{aligned}
& K(\mu) \alpha^{\prime}(\mu)-L(\mu) \beta^{\prime}(\mu)+M(\mu)=0, \\
& L(\mu) \alpha^{\prime}(\mu)+K(\mu) \beta^{\prime}(\mu)+N(\mu)=0,
\end{aligned}
$$

where:

$$
\begin{aligned}
& K(\mu)=3 \alpha^{2}(\mu)+2 b_{1}(\mu) \alpha(\mu)+b_{2}(\mu)-3 \beta^{2}(\mu) \\
& L(\mu)=6 \alpha(\mu) \beta(\mu)+2 b_{1}(\mu) \beta(\mu) \\
& M(\mu)=b_{1}^{\prime}(\mu)\left(\alpha^{2}(\mu)-\beta^{2}(\mu)\right)+b_{2}^{\prime}(\mu) \alpha(\mu)+b_{3}^{\prime}(\mu) \\
& N(\mu)=2 b_{1}^{\prime}(\mu) \alpha(\mu) \beta(\mu)+b_{2}^{\prime}(\mu) \beta(\mu)
\end{aligned}
$$

Since $K\left(\mu_{1}^{*}\right) M\left(\mu_{1}^{*}\right)+L\left(\mu_{1}^{*}\right) N\left(\mu_{1}^{*}\right) \neq 0$, we have:

$$
\begin{aligned}
& \operatorname{Re}\left[\frac{d r_{i}}{d \mu}\right]_{\mu=\mu_{1}^{*}}=\left.\alpha^{\prime}(\mu)\right|_{\mu=\mu_{1}^{*}} \\
& =-\left.\frac{K(\mu) M(\mu)+L(\mu) N(\mu)}{K^{2}(\mu)+L^{2}(\mu)}\right|_{\mu=\mu_{1}^{*}} \neq 0, i=2,3
\end{aligned}
$$

and $r_{4}\left(\mu_{1}^{*}\right)=-b_{1}\left(\mu_{1}^{*}\right) \neq 0$.

Hence there is a Hopf bifurcation at $\mu=\mu_{1}^{*}$. We have the following result:

\section{Theorem 13}

Suppose $R_{1}$ holds. Then the system (29) undergoes a Hopf bifurcation when $\mu$ passes through $\mu_{1}^{*}$ emanating from the steady state $E_{6}\left(\tilde{s}, \tilde{x}_{1}, 0, \tilde{y}\right)$ leading to periodic solutions trajectories for either $\mu>\mu_{1}^{*}$ (supercritical bifurcation), or $\mu<\mu_{1}^{*} \quad$ (sub-criticalbifurcation) or at $\mu=\mu_{1}^{*}$.

Next, Hopf bifurcation at $E_{8}\left(\lambda_{2}, \bar{x}_{1}, \bar{x}_{2}, \bar{y} ; \mu\right)$ :

The Jacobian matrix corresponding to $E_{8}\left(\lambda_{2}, \bar{x}_{1}, \bar{x}_{2}, \bar{y} ; \mu\right)$ is given by:

$$
J_{\mu}\left(E_{8}\right)=\left[n_{i j}\right]_{4 \times 4}
$$

where, $J_{\mu}\left(E_{8}\right)$ and its nonzero elements $n_{i j}$ are defined as in (14) and (15) with $p_{1}(\tilde{s})$ and $p_{1}^{\prime}(\tilde{s})$ replaced by $p_{1}(\tilde{s} ; \mu)$ and $p_{1}{ }^{\prime}(\tilde{s} ; \mu)$ respectively.

The characteristic equation corresponding to $J_{\mu}\left(E_{8}\right)$ obeys:

$r^{4}+d_{1} r^{3}+d_{2} r^{2}+d_{3} r+d_{4}=0$ 
where the coefficients $d_{i}=\bar{a}_{i}, i=1,2,3,4$ are defined as in (16).

By the Routh-Hurwitz criteria, all the roots of (37) will have negative real parts if:

$$
\begin{aligned}
& R_{3}: d_{1}>0, d_{3}>0, d_{4}>0, d_{1} d_{2}>d_{3} \\
& \text { and } R_{4}: d_{1} d_{2} d_{3}>d_{3}{ }^{2}+d_{4} d_{1}{ }^{2} .
\end{aligned}
$$

It is required for a Hopf bifurcation that either $R_{3}$ or $R_{4}$ must be violated. Suppose $d_{i}>0, i=1,2,3,4$ such that:

- $\quad d_{1} d_{2}>d_{3}$

- $R_{4}$ is violated such that $d_{1} d_{2} d_{3}=d_{3}^{2}+d_{4} d_{1}^{2}$ $\Leftrightarrow d_{2} d_{3}=\frac{d_{3}^{2}}{d_{1}}+d_{1} d_{4}>d_{4}$

In factor form, the Equation 37 can be written as:

$$
\begin{aligned}
& \left(r^{2}+\theta_{1}\right)\left(r+\theta_{2}\right)\left(r+\theta_{3}\right)=0 \\
& \theta_{i}>0, i=1,2,3
\end{aligned}
$$

where:

$$
\begin{aligned}
& d_{1}=\theta_{2}+\theta_{3}, d_{2}=\theta_{2} \theta_{3}+\theta_{1} \\
& d_{3}=\theta_{1}\left(\theta_{2}+\theta_{3}\right) \text { and } d_{4}=\theta_{1} \theta_{2} \theta_{3}
\end{aligned}
$$
roots:

In particular, Equation 37 leads the following set of

$$
\sigma\left(J_{\mu}\left(E_{8}\right)\right)=\left\{i \sqrt{\theta_{1}},-i \sqrt{\theta_{1}},-\theta_{2},-\theta_{3}\right\}
$$

where, $\theta_{i}, i=1,2,3$ are solution of (38).

It shows that Equation 37 has two pure imaginary roots for some value of $\mu$, say $\mu_{2}^{*}$. Therefore, there is an open interval containing $\mu_{2}^{*}$, say $\left(\mu_{2}^{*}-\varepsilon, \mu_{2}^{*}+\varepsilon\right)$ for some $\varepsilon>0$, the equation (37) cannot have positive real roots. But for $\mu \in\left(\mu_{2}^{*}-\varepsilon, \mu_{2}^{*}+\varepsilon\right)$, the roots are in general of the form:

$$
\begin{aligned}
& r_{1}(\mu)=\alpha(\mu)+i \beta(\mu), r_{2}(\mu)=\alpha(\mu)-i \beta(\mu), \\
& r_{3}(\mu)=-\theta_{2}(\mu) \text { and } r_{4}(\mu)=-\theta_{3}
\end{aligned}
$$

Now we apply Hopf's transversality condition (34) so as to obtain the desired conditions for Hopf bifurcation to occur for system (29). Substituting $r_{1}(\mu)=\alpha(\mu)+i \beta(\mu)$ and $r_{2}(\mu)=\alpha(\mu)-i \beta(\mu)$ into (37) and computing the derivatives with respect to $\mu$, we get:

$$
\begin{gathered}
\bar{K}(\mu) \alpha^{\prime}(\mu)-\bar{L}(\mu) \beta^{\prime}(\mu)+\bar{M}(\mu)=0, \\
\bar{L}(\mu) \alpha^{\prime}(\mu)+\bar{K}(\mu) \beta^{\prime}(\mu)+\bar{N}(\mu)=0,
\end{gathered}
$$

where:

$$
\begin{aligned}
& \bar{K}(\mu)=4 \alpha\left(\alpha^{2}-\beta^{2}\right)-8 \alpha \beta^{2}+3 d_{1}\left(\alpha^{2}-\beta^{2}\right)+2 d_{2} \alpha+d_{3} \\
& \bar{L}(\mu)=4 \beta\left(\alpha^{2}-\beta^{2}\right)+8 \alpha^{2} \beta+6 d_{1} \alpha \beta+2 d_{2} \beta \\
& \bar{M}(\mu)=d_{1}^{\prime}\left[\alpha\left(\alpha^{2}-\beta^{2}\right)-2 \alpha \beta^{2}\right]+d_{2}^{\prime}\left(\alpha^{2}-\beta^{2}\right)+d_{3}^{\prime} \alpha+d_{4}^{\prime} \\
& \bar{N}(\mu)=d_{1}^{\prime}\left[\beta\left(\alpha^{2}-\beta^{2}\right)+2 \alpha^{2} \beta\right]+2 d_{2}^{\prime} \alpha \beta+d_{3}^{\prime} \beta
\end{aligned}
$$

Since $\bar{K}\left(\mu_{2}^{*}\right) \bar{M}\left(\mu_{2}^{*}\right)+\bar{L}\left(\mu_{2}^{*}\right) \bar{N}\left(\mu_{2}^{*}\right) \neq 0$, we have:

$$
\begin{aligned}
& \operatorname{Re}\left[\frac{d r_{i}}{d \mu}\right]_{\mu=\mu_{2}^{*}}=\left.\alpha^{\prime}(\mu)\right|_{\mu=\mu_{2}^{*}} \\
& =-\left.\frac{\bar{K}(\mu) \bar{M}(\mu)+\bar{L}(\mu) \bar{N}(\mu)}{\bar{K}^{2}(\mu)+\bar{L}^{2}(\mu)}\right|_{\mu=\mu_{2}^{*}} \neq 0, i=1,2
\end{aligned}
$$

and $r_{3}\left(\mu_{2}^{*}\right)=-\theta_{2}\left(\mu_{2}^{*}\right) \neq 0, \quad r_{4}\left(\mu_{2}^{*}\right)=-\theta_{3}\left(\mu_{2}^{*}\right) \neq 0$. Hence there is a Hopf bifurcation at $\mu=\mu_{2}^{*}$. We summarize above discussion in the following result.

\section{Theorem 14}

Suppose (i) $E_{8}\left(\lambda_{2}, \bar{x}_{1}, \bar{x}_{2}, \bar{y}\right)$ exists (ii) $d_{i}>0, i=1,2,3,4$, $d_{1} d_{2}>d_{3}$ and $R_{4}$ is violated. Then system (29) exhibits a Hopf bifurcation in the first orthant, leading to a family of periodic solutions trajectories that bifurcate from $E_{8}$ when $\mu$ varies in a small neighborhood of $\mu=\mu_{2}^{*}$.

\section{Discussion and Conclusion}

We showed a detailed mathematical analysis of a classical food web model describing three species interaction competing for a single nutrient in which one of the competitors predates on one of the other competitors. We assumed general monotone response functions for the nutrient uptake of the competitors and growth rate for predator. The considered model is more general and its analysis extends the works of the system (El-Owaidy and Moniem, 2003; Li and Kuang, 2000; Butler and Wolkowicz, 1986; Wolkowicz, 2006). We proved that the trajectories of system (2) are bounded for all future time. We showed that the equilibria for system (2) are LAS if exist by using Routh-Hurwitz criterion. We found that all the species cannot persist if break-even concentrations of the preys are close to one. This happens when $E_{1}$ is a global attractor of system (2). We proved that if exists the equilibria $E_{2}$ and $E_{6}$ are globally asymptotically stable by using Liapunov functions as in (Nani and Freedman, 2000) showing that the predator will be die out in the chemostat whatever be the initial concentrations of prey and predator. Next, when $E_{8}$ exists then all the species (predator-preys) coexist, 
that is, the system (2) is persistent uniformly and circumvented the conservation principle. We applied the similar technique as in (Freedman and Waltman, 1984) and presented that the system (2) passes though a Hopf bifurcation at $E_{6}$ leading to periodic solutions. Finally, we established theoretical criteria for which the system (2) undergoes a Hopf Andronov-Poincare bifurcation at the equilibrium point $E_{8}$ and possesses a family of periodic solutions that bifurcates from $E_{8}$. Therefore, we can conclude that the results obtained in this study partially generalize and improve to the works done by (Butler and Wolkowicz, 1986; ElOwaidy and Moniem, 2003; Li and Kuang, 2000; Nasrin and Rana, 2011; Wolkowicz, 2006).

\section{Acknowledgment}

The author would like to thank the referees for their valuable comments that help to improve the manuscript.

\section{Ethics}

This article is original and contains unpublished material. The author confirms that he has read and approved the manuscript and no ethical issues involved.

\section{References}

Alhumazi, K. and A. Ajbar, 2005. Dynamics of predatorprey interactions in continuous cultures. Eng. Life Sci., 5: 139-147. DOI: 10.1002/elsc. 200420062

Aris, R. and A.E. Humphrey, 1977. Dynamics of a chemostat in which two organisms compete for a common substrate. Biotechnol. Bioeng., 19: 1375-1386. DOI: 10.1002/bit.260190910

Butler, G.J. and G.S.K. Wolkowicz, 1985. A mathematical model of the chemostat with a general class of functions describing nutrient uptake. SIAM J. Appl. Math., 45: 138-151. DOI: $10.1137 / 0145006$

Butler, G.J. and G.S.K. Wolkowicz, 1986. Predatormediated competition in the chemostat. J. Math. Biol., 24: 167-191. DOI: 10.1007/BF00275997

Butler, G.J., S.B. Hsu and P. Waltman, 1983. Coexistence of competing predators in a chemostat. J. Math. Biol., 17: 133-151. DOI: 10.1007/BF00305755

Butler, G.J., H.I. Freedman and P. Waltman, 1986. Uniformly persistent systems. Proc. Amer. Math. Soc., 96: 425-430. DOI: $10.1090 / \mathrm{S} 0002-9939-1986-0822433-4$

Boer, M.P., B.W. Kooi and S.A.L.M. Koojiman, 1998. Food chain dynamics in the chemostat. Math. Biosci., 150: 43-62. DOI: 10.1016/S0025-5564(98)00010-8

Chiu, C.H. and S.B. Hsu, 1998. Extinction of toppredator in a three-level food-chain model. J. Math. Biol., 37: 372-380. DOI: 10.1007/s002850050134
Drake, J.F. and H.M. Tsuchiya, 1976. Predation of Escherichia coli by Colpoda steinii. Applied Envir. Microbiol. 31: 870-874.

El-Owaidy, H.M. and A.A. Moniem, 2003. On food chain in a chemostat with distinct removal rates. Applied Math. E-Notes, 3: 183-191.

El-Sheikh, M.M.A. and S.A.A. Mahrouf, 2005. Stability and bifurcation of a simple food chain in a chemostat with removal rates. Chaos Solitons Fractals, 23: 1475-1489. DOI: 10.1016/j.chaos.2004.06.079

Freedman H.I. and S. Ruan, 1992. Hopf bifurcation in three-species food chain models with group defense. Math. Biosci., 111: 73-87. DOI: 10.1016/0025-5564(92)90079-C

Freedman, H.I. and P. Waltman, 1977. Mathematical analysis of some three-species food-chain models. Math. Biosci., 33: 257-276. DOI: 10.1016/0025-5564(77)90142-0

Freedman, H.I. and P. Waltman, 1984. Persistence in models of three interacting predator-prey populations. Math. Biosci., 68: 213-31. DOI: 10.1016/0025-5564(84)90032-4

Huang, X. and L. Zhu, 2005. A three dimensional chemostat with quadratic yields. J. Math. Chem., 38: 575-588. DOI: 10.1007/s10910-005-6908-0

Hsu, S.B., S. Hubbell and P. Waltman, 1977. A mathematical theory for single-nutrient competition in continuous cultures of micro-organisms. SIAM J. Applied Math., 32: 366-383. DOI: 10.1137/0132030

Hastings, A. and T. Powell, 1991. Chaos in a threespecies food chain. Ecology, 72: 896-903. DOI: $10.2307 / 1940591$

Jost, J.L., J.F. Drake, H.M. Tsuchiya and A.G. Fredrickson, 1973. Microbial food chains and food webs. J. Theor. Biol., 41: 461-484. DOI: 10.1016/0022-5193(73)90056-8

Jost, J.L., J.F. Drake, A.G. Fredrickson and H.M. Tsuchiya, 1973. Interactions of Tetrahymena pyriformis, Escherichia coli, Azotobacter vinelandii and glucose in a minimal medium. J. Bacteriol., 113: 834-840. PMID: 4632323

Klebanoff, A. and A. Hastings, 1994. Chaos in three species food chains. J. Math. Biol., 32: 427-451. DOI: $10.1007 / \mathrm{BF} 00160167$

Li, B. and Y. Kuang, 2000. Simple food chain in a chemostat with distinct removal rates. J. Math. Anal. Applic., 242: 75-92. DOI: 10.1006/jmaa.1999.6655

Li, B., 1999. Global asymptotic behavior of the chemostat: General response functions and different removal rates. SIAM J. Applied Math., 59: 411-422. DOI: $10.1137 /$ S003613999631100X

LaSalle, J., S. Lefchetz, 1961. Stability by Liapunov's Direct Method. 1st Edn., Academic Press, New York, pp: 134. 
Marsden, J.E. and M. Mckracken, 1976. The Hopf Bifurcation and its Applications. 1st Edn., SpringerVerlag, New York, ISBN-10: 3540902007, pp: 408.

Nani, F. and H.I. Freedman, 2000. A mathematical model of cancer treatment by immunotherapy. Math. Biosci., 163: 159-99.

DOI: 10.1016/S0025-5564(99)00058-9

Nasrin, F. and S.M.S Rana, 2011. Three species food web in a chemostat. Int. J. Appl. Sci. Eng., 9, 4: 301-313.

Pavlou, S., 1985. Dynamics of a chemostat in which one microbial population feeds on another. Biotechnol. Bioeng., 27: 1525-1532. DOI: $10.1002 /$ bit.260271102

Ruan, S. and H.I. Freedman, 1991. Persistence in threespecies food chain models with group defense. Math. Biosci. 107: 111-125. DOI: 10.1016/0025-5564(91)90074-S

Smith, H.L. and P. Waltman, 1995. The Theory of the Chemostat: Dynamics of Microbial Competition. 1st Edn., Cambridge University Press, Cambridge, ISBN-10: 0521470277, pp: 313.

Al-Sheikh, S.A., 2008. The dynamics of a tri-trophic food chain in the chemostat. Int. J. Pure. Applied Math., 47: 101-111.
Tsuchiya, H.M., S.F. Drake, J.L. Jost and A.G. Fredrickson, 1972. Predator-Prey Interactions of Dictyostelium discoideum and Escherichia coli in Continuous Culture. J. Bacteriol., 110: 1147-1153.

Vayenas, D.V. and S. Pavlou, 1999. Chaotic dynamics of a food web in a chemostat. Math. Biosci., 162: 6984. DOI: 10.1016/S0025-5564(99)00044-9

Wolkowicz, G.S.K., 2006. Interpretation of the generalized asymmetric May-Leonard model of three species competition as a food web in a chemostat. Fields Instit. Commun., 48: 279-289.

Wolkowicz, G.S.K. and Z. Lu, 1992. Global dynamics of a mathematical model of competition in the chemostat: General response functions and differential death rates. SIAM J. Applied Math., 52: 222-233. DOI: $10.1137 / 0152012$

Waltman, P., S.P. Hubbel and S.B. Hsu, 1980. Theoretical and Experimental Investigation of Microbial Competition in Continuous Culture. Modeling and Differential Equations in Biology, Burton, T. (Ed.), pp: 107-192.

Zhu, H., S.A. Campbell and G.S.K. Wolkowicz, 2002. Bifurcation analysis of a predator-prey system with nonmonotonic functional response. SIAM J. Applied Math., 63: 636-682. 